\title{
Organometallic Synthesis and Spectroscopic Characterization of Manganese-Doped CdSe Nanocrystals
}

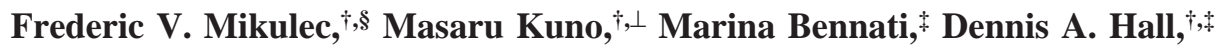 \\ Robert G. Griffin, ${ }^{\dagger,}$ and Moungi G. Bawendi*, ${ }^{\dagger}$
}

Contribution from the Department of Chemistry and the Center for Magnetic Resonance, Francis Bitter
Magnet Laboratory, Massachusetts Institute of Technology, Cambridge, Massachusetts 02139

Received April 19, 1999. Revised Manuscript Received December 7, 1999

\begin{abstract}
The synthesis of II-VI semiconductor nanocrystals doped with transition metals has proved to be particularly difficult. In the case of CdSe quantum dots (QDs) produced via high-temperature pyrolysis in trioctylphosphine oxide (TOPO), specially designed precursors used in this study appear to be necessary to successfully incorporate low levels of $\mathrm{Mn}$. A simple etching experiment and electron paramagnetic resonance (EPR) measurements reveal that most of the dopant atoms reside in the surface layers of the inorganic lattice. The dopant dramatically affects ${ }^{113} \mathrm{Cd}$ magic angle spinning (MAS) nuclear magnetic resonance NMR spectra; the observed paramagnetic shift and decreased longitudinal relaxation time are consistent with Mn incorporated in the QDs. Paramagnetic atoms in QDs generate large effective magnetic fields, which implies that magnetooptical experiments can be performed simply by doping. Results from fluorescence line narrowing (FLN) studies on Mn-doped CdSe QDs mirror previous findings on undoped QDs in an external magnetic field. Experimental fitting of photoluminescence excitation (PLE) spectra of doped QDs reveals that the effective absorption line shape contains a new feature that is believed to be a previously unobserved, but theoretically predicted, optically dark fine structure state.
\end{abstract}

\section{Introduction}

Although there is a large body of literature concerning transition metal doped semiconductors, ${ }^{1}$ also called diluted magnetic semiconductors (DMS), and there has been a flurry of activity investigating semiconductor quantum dots ${ }^{2}$ (QDs), it is only recently that there have been reports describing research occurring at the intersection of these two fields. ${ }^{3}$ Of the work detailing the synthesis of DMS QDs, the majority rely on photoluminescence (PL) spectroscopy to determine if the materials have been successfully doped: below band gap energy emission at the wavelength corresponding to the bulk material is the primary litmus test for doped nanocrystals. However, this emission only implies that electronic coupling exists between

\footnotetext{
* Author to whom correspondence should be sent.

Department of Chemistry, MIT.

$\doteqdot$ Center for Magnetic Resonance, MIT.

$\S$ Present address: Department of Chemistry and Biochemistry, UCSD, La Jolla, CA 92093-0358.

${ }^{\perp}$ Present address: JILA, University of Colorado, Boulder, CO 80308

(1) Furdyna, J. K. J. Appl. Phys. 1988, 64, R29-R64.

(2) Alivisatos, A. P. J. Phys. Chem. 1996, 100, 13226-13239.

(3) (a) Wang, Y.; Herron, N.; Moller, K.; Bein, T. Solid State Commun. 1991, 77, 33-38. (b) Dhingra, S.; Kim, K.-W.; Kanatzidis, M. G. Mater. Res. Soc. Symp. Proc. 1991, 204, 163-168. (c) Kim, K.-W.; Cowen, J. A.; Dhingra, S.; Kanatzidis, M. G. Mater. Res. Soc. Symp. Proc. 1992, 272, 27-33. (d) Yanata, K.; Suzuki, K.; Oka, Y. J. Appl. Phys. 1993, 73, 45954598. (e) Bhargava, R. N.; Gallagher, D.; Hong, X.; Nurmikko, A. Phys. Rev. Lett. 1994, 72, 416-419. (f) Bhargava, R. N.; Gallagher, D.; Welker, T. J. Lumin. 1994, 60 and 61, 275-280. (g) Gallagher, D.; Heady, W. E.; Racz, J. M.; Bhargava, R. N. J. Cryst. Growth 1994, 138, 970-975. (h) Yanata, K.; Oka, Y. Jpn. J. Appl. Phys. 1995, Suppl. 34-1, 164-166. (i) Bandaranayake, R. J.; Smith, M.; Lin, J. Y.; Jiang, H. X.; Sorensen, C. M. IEEE Trans. Magn. 1994, 30, 4930-4932. (j) Sooklal, K.; Cullum, B. S.; Angel, S. M.; Murphy, C. J. J. Phys. Chem. 1996, 100, 45514555. (k) Levy, L.; Hochepied, J. F.; Pileni, M. P. J. Phys. Chem. 1996, 100, 18322-18326. (1) Counio, G.; Esnouf, S.; Gacoin, T.; Boilot, J.-P. J. Phys. Chem. 1996 100, 20021-20026. (m) Levy, L.; Feltin, N.; Ingert, D.; Pileni, M. P. J. Phys. Chem. B 1997, 101, 9153-9160.
}

the nanocrystal and the Mn excited states. Although it is then usually inferred that the Mn dopant must be inside the QD lattice, the structural evidence supporting these claims is often minimal. An exception is work reported by Kanatzidis and coworkers, ${ }^{3 \mathrm{c}}$ which presents nanocrystalline $\mathrm{Cd}_{1-x} \mathrm{Mn}_{x}$ Se powder $\mathrm{X}$-ray diffraction patterns displaying with textbook clarity the linear dependence of the lattice constant with $x$. Although their method is powerful because it allows $x$ to be varied continuously from 0 to 1 , the material is too large (>200 $\AA$ ) to exhibit quantum confinement.

A recent report from Ladizhansky and co-workers concludes that the dopant in their CdMnS samples, prepared by the popular coprecipitation method, is either in the solvent/ligand matrix or bound to the surface of the particles. ${ }^{4}$ In light of these results, any thorough characterization of doped QDs must include convincing structural evidence, or numerous purification steps and control experiments, that determines the location of the dopant atoms from among a number of possibilities: trapped in the matrix containing the sample, bound to the protecting surface ligands, adsorbed onto the surface of the particles, doped randomly within the inorganic lattice, etc.

By incorporating manganese into the QD one can investigate the effects of a paramagnetic dopant, and the effective magnetic field it generates, on the semiconductor properties. Here we are particularly concerned with how the dopant affects the characteristics of the quantum dot optical properties. The paramagnetic impurity provides a means of coupling the optical and magnetic properties of these materials via the $\mathrm{sp}-\mathrm{d}$ exchange interaction. This strong interaction between the carriers of the semiconductor and the spin of the dopant is well-known in bulk DMS; it is at the origin of the unique properties of these materials, including

(4) Ladizhansky, V.; Hodes, G.; Vega, S. J. Phys. Chem. B 1998, 102, 8505-8509. 
giant Faraday rotation effects near the band edge. ${ }^{5}$ The preparation of doped QDs should allow the study of the effects of threedimensional quantum confinement on well-known bulk DMS properties. A report by Awschalom and co-workers ${ }^{6}$ of changes in the magnetic behavior of thin CdMnTe films as the layer thickness is reduced from 86 to $18 \AA$ suggests that quantum confinement should affect the manganese-manganese interaction (the $\mathrm{d}-\mathrm{d}$ exchange) as well. Manganese-doped nanocrystals are likely to display properties which have not been observed in either bulk DMS or undoped semiconductor QDs.

In this paper we describe the use of the single source manganese- and chalcogen-containing organometallic complex $\mathrm{Mn}_{2}(\mu-\mathrm{SeMe})_{2}(\mathrm{CO})_{8}$ to prepare $\mathrm{Cd}_{1-x} \mathrm{Mn}_{x} \mathrm{Se} \mathrm{QDs}$ of various sizes. Simpler precursors containing only manganese were ineffective dopants when incorporated into the trioctylphosphine oxide (TOPO) method for making CdSe QDs. Extensive surface ligand exchange was performed to ensure that our quantitative measurements detected only manganese ions incorporated into the lattice, rather than species loosely bound to the nanocrystal surface. For $\mathrm{Cd}_{1-x} \mathrm{Mn}_{x} \mathrm{Se} \mathrm{QDs}$, there is good agreement between manganese concentrations determined by wavelength dispersive $\mathrm{X}$-ray spectroscopy (WDS) and results from electron paramagnetic resonance (EPR) measurements. Magic angle spinning solid-state ${ }^{113} \mathrm{Cd}$ nuclear magnetic resonance (NMR) spectra of doped QDs display the paramagnetic shift and decreased longitudinal relaxation times reported for the corresponding bulk material. We study the effect on the optical properties via fluorescence line narrowing (FLN) and photoluminescence excitation (PLE) experiments. By fitting the resulting spectra we extract emission and absorption line shapes where inhomogeneites associated with the residual size distribution have been significantly reduced. The extracted emission line shapes display trends which had previously been seen in undoped nanocrystals under the influence of an external magnetic field. The corresponding extracted absorption line shapes of the doped QDs can be viewed as excitation spectra in the presence of a magnetic field. Such an experiment has not been performed before this point, and it reveals new structure in the absorption line shape. This new feature can be explained in the context of a dark exciton model of the band edge luminescence. The convergence of results from doped QDs and undoped QDs in a magnetic field confirms that transition metal doping provides a powerful and simple means of performing high magnetic field experiments without an external magnet.

\section{Experimental Section}

General. Unless otherwise noted, all reactions were carried out in a dry nitrogen atmosphere. Solvents used in the precursor synthesis were either anhydrous grade or dried via distillation over a suitable drying agent. HPLC grade solvents used in QD purification were purchased from EM Science. Methyllithium (1.4 M in ether) was purchased from Aldrich. Trioctylphosphine (TOP, 95\%) and tripyrrolidinophosphine oxide $(\geq 98 \%)$ were purchased from Fluka. Selenium shot, tellurium powder, tricarbonyl methylcyclopentadienylmanganese (TCPMn), and manganese(II) cyclohexanebutyrate (an atomic absorption spectroscopy standard) were purchased from Alfa. Trioctylphosphine oxide (TOPO, 90\%), $\mathrm{CdMe}_{2}(99+\%), \mathrm{Mn}_{2}(\mathrm{CO})_{10}$, and $\mathrm{Mn}-$ $(\mathrm{CO})_{5} \mathrm{Br}$ were obtained from Strem. All chemicals were used as 191.

(5) Oh, E.; Ramdas, A. K.; Furdyna, J. K. J. Lumin. 1992, 52, 183-

(6) Awschalom, D. D.; Hong, J. M.; Chang, L. L.; Grinstein, G. Phys. Rev. Lett. 1987, 59, 1733-1736.

(7) (a) Closson, R. D.; Kozikowski, J.; Coffield, T. H. J. Org. Chem. 1957, 22, 598. (b) Bruce, M. I.; Liddell, M. J.; Pain, G. N. Inorg. Synth. 1989, 26, 171-180. (c) Steigerwald, M. L.; Rice, C. E. J. Am. Chem. Soc. 1988, 110, 4228-4231. received, except TOPO, which was dried under vacuum $(\sim 0.5$ Torr $)$ at $180{ }^{\circ} \mathrm{C}$ for $1 \mathrm{~h}$, and $\mathrm{CdMe}_{2}$, which was passed through a $0.2 \mu \mathrm{m}$ PTFE membrane syringe filter.

Precursors. The precursors, $\mathrm{MnMe}_{2}, \mathrm{Mn}(\mathrm{CO})_{5} \mathrm{Me}$, and $\left[\mathrm{MnTe}(\mathrm{CO})_{3^{-}}\right.$ $\left.\left(\mathrm{PEt}_{3}\right)_{2}\right]_{2}$ were synthesized according to literature methods. ${ }^{7}$ These compounds were used in early attempts to dope the nanocrystals before settling on the following single source MnSe precursor.

$\mathrm{Mn}_{2}(\mu \text {-SeMe })_{2}(\mathrm{CO})_{8}$ was synthesized by adapting Coleman's procedure for making the analogous tellurium compound. ${ }^{8}$ In the preparation, $13 \mathrm{~mL}(18 \mathrm{mmol})$ of a $1.4 \mathrm{M}$ solution of methyllithium in ether was added dropwise to a stirring suspension of $1.46 \mathrm{~g}(18.5 \mathrm{mmol})$ of selenium powder in $100 \mathrm{~mL}$ of THF. The resulting clear (slightly black) mixture was stirred at room temperature for $15 \mathrm{~min}$. A solution consisting of $5.01 \mathrm{~g}(18.2 \mathrm{mmol})$ of $\mathrm{Mn}(\mathrm{CO})_{5} \mathrm{Br}$ in $50 \mathrm{~mL}$ of THF was then added dropwise to the mixture. A dark red solution was obtained and allowed to stir for $15 \mathrm{~min}$. The solvent was removed under vacuum, leaving an oil. That oil was extracted with boiling hexane or pentane. Removal of the solvent under vacuum left an orange-brown solid (75\% yield) whose IR spectrum (carbonyl region) and ${ }^{1} \mathrm{H}$ NMR spectrum match literature values for $\mathrm{Mn}_{2}(\mu-\mathrm{SeMe})_{2}(\mathrm{CO})_{8} .{ }^{9}$

Undoped CdSe quantum dots were synthesized according to previously described literature methods. ${ }^{10}$ Doped nanocrystals were prepared in a similar fashion by introducing a small amount of precursor (2$200 \mathrm{mg}$ ) to the TOP injection solution. The manganese precursor was dissolved by sonicating the suspension for $10-30 \mathrm{~min}$ to give a clear, red-brown solution. $\mathrm{CdMe}_{2}(200 \mu \mathrm{L}, 2.78 \mathrm{mmol})$ and $3 \mathrm{~mL}(3 \mathrm{mmol})$ of $1 \mathrm{M}$ TOPSe were then added and the solution was mixed and then injected into $30 \mathrm{~g}$ of dry, stirring TOPO at $350{ }^{\circ} \mathrm{C}$. For example, the injection solution used to prepare the FLN/PLE sample consisted of $200 \mu \mathrm{L}$ of $\mathrm{CdMe}_{2}, 3 \mathrm{~mL}$ of $1 \mathrm{M}$ TOPSe, $30 \mathrm{mg}$ of $\mathrm{Mn}_{2}(\mu-\mathrm{SeMe})_{2}-$ $(\mathrm{CO})_{8}$, and $16 \mathrm{~mL}$ of TOP.

Upon injecting, the reaction temperature fell to $\sim 260{ }^{\circ} \mathrm{C}$ and the solution immediately turned yellow/orange. The $\lambda_{\max }$ of the initial UV/ visible absorption was in the range of 480 to $510 \mathrm{~nm}$, indicating the presence of small 10-15 ̊ radius CdSe QDs. Heating was restored to the reaction flask and the temperature raised to $300{ }^{\circ} \mathrm{C}$ in 5 to $10 \mathrm{deg}$ $\mathrm{C}$ increments to promote steady growth of the nanocrystals. The growth was monitored by the UV/visible absorbance of the first excited state. Doping levels can be increased by dropwise addition of more injection solution to the hot reaction mixture.

Once a sample reached the desired size, the temperature was lowered to $60{ }^{\circ} \mathrm{C}$ and $15 \mathrm{~mL}$ of butanol was added to the growth solution to prevent solidification. All subsequent manipulations of the QDs were performed in air. The nanocrystals were isolated from solution by size selective precipitation using methanol. ${ }^{10 a, 11}$ Surface ligand exchange was performed as previously described. ${ }^{10 \mathrm{~b}}$

Electron Paramagnetic Resonance (EPR). Room temperature (300 $\mathrm{K}, 9.77 \mathrm{GHz})$ and low-temperature $(4.2 \mathrm{~K}, 9.44 \mathrm{GHz})$ EPR spectra were obtained using a Bruker ESP 300 instrument. Microwave powers employed were below saturation levels. Quantitative standards were prepared by adding known amounts of a manganese(II) cyclohexanebutyrate stock solution to undoped CdSe QDs in toluene. The concentrations of nanocrystals in the standard and the unknown were approximately equal. Areas related to the abundance of each compound were calculated by double integration of the experimental first derivative spectra. The amount of manganese added to the reaction which ended up doping the nanocrystals was generally $1 \%$ (at.) or less.

Wavelength Dispersive X-ray Spectroscopy (WDS). A JEOL SEM 733 electron microprobe operating at $15 \mathrm{kV}$ was used to determine manganese concentrations. The takeoff angle to the detector was $40^{\circ}$ and the magnification was $1000 \times$. Samples were prepared by precipitating TOPO/TOPSe capped nanocrystals from solution and washing the material three times with methanol. Following the third wash, the

(8) Coleman, A. P.; Dickson, R. S.; Deacon, G. B.; Fallon, G. D.; Ke, M.; McGregor, K.; West, B. O. Polyhedron 1994, 13, 1277-1290.

(9) Welcman, N.; Rot, I. J. Chem. Soc. 1965, 7515-7516.

(10) (a) Murray, C. B.; Norris, D. J.; Bawendi, M. G. J. Am. Chem. Soc. 1993, 115, 8706-8715. (b) Kuno, M.; Lee, J. K.; Dabbousi, B. O.; Mikulec, F. V.; Bawendi, M. G. J. Chem. Phys. 1997, 106, 9869-9882.

(11) Chemseddine, A.; Weller, H. Ber. Bunsen-Ges. Phys. Chem. 1993, 97, 636-637. 
QDs were dissolved in a small amount of pyridine. One drop of the concentrated pyridine solution was placed on a silicon (100) wafer and dried under vacuum at $80{ }^{\circ} \mathrm{C}$ for $24 \mathrm{~h}$. The films were later coated with a thin layer of amorphous carbon to prevent charging effects under the electron beam.

Solid-State NMR. Magic angle spinning (MAS) NMR experiments were performed using a home-built $211 \mathrm{MHz}$ NMR spectrometer (47 $\mathrm{MHz}$ for ${ }^{113} \mathrm{Cd}$ ) and a custom-designed, two-channel transmission line probe. The ${ }^{113} \mathrm{Cd} \pi / 2$ pulse width was $5 \mu$ s. Hahn echo pulse sequences $(\pi / 2-\tau-\pi-\tau-$ Acq) were used for all spectra, with the interpulse delay $\tau$ equal to the rotor period. A $5 \mathrm{~mm}$ Chemagnetics spinning system (Otsuka Electronics USA) was used for MAS, with spinning frequencies of the samples varied from 6.5 to $9 \mathrm{kHz}$. Spin-lattice relaxation times ( $T_{1}$ values) were estimated from the recycle delays.

Optical Spectroscopy. Variable-temperature fluorescence line narrowing (FLN) and photoluminescence excitation (PLE) experiments were conducted on doped and undoped QDs using a SPEX Fluorolog-2 spectrofluorometer. The entrance and exit slits of both excitation and emission spectrometers were set to $125 \mu \mathrm{m}$ to give an optical resolution of $0.15-0.2 \mathrm{~nm}$. Samples were prepared by loading hexane solutions of TOPO/TOPSe capped nanocrystals between two sapphire flats separated by a $0.5 \mathrm{~mm}$ Teflon spacer. The absorbance of the lowest energy feature in all samples was kept below 1.0 to minimize reabsorption of the luminescence.

The FLN experiment was conducted by choosing five energies over the full luminescence profile of the nanocrystals. The sample was then excited sequentially at each of these energies and the resulting luminescence detected through the emission spectrometer/PMT. The PLE experiment followed in a similar fashion. In this case, though, the detector was fixed at a particular wavelength (energy) and the excitation spectrometer was scanned to the blue of this position to reproduce the absorption profile of the nanocrystals. The detector energy was determined by choosing the peak of the zero phonon line (ZPL) in each of the five emission scans. In this manner the absorption and emission of five "effective" QD sizes were determined using one sample. Variable temperatures were achieved using a Janis helium vapor flow cryostat.

\section{Results and Discussion}

Testing Mn Precursors. The use of organometallic manganese complexes as precursors in the chemical vapor deposition of DMS films has been well documented. ${ }^{12}$ Such compounds are attractive candidates for nanocrystalline DMS synthesis because they are compatible with solvents used in the present method and they decompose cleanly at low temperatures. For example, TCPMn decomposes at $300-500{ }^{\circ} \mathrm{C}$ and $\mathrm{Mn}(\mathrm{CO})_{5} \mathrm{Me}$ at $100{ }^{\circ} \mathrm{C}$. These precursors are soluble in TOP and are easily dispersed in the standard injection solution at concentrations up to $\sim 10 \mathrm{mg} / \mathrm{mL}$. This low concentration of precursors does not greatly disrupt growth of the host CdSe lattice, allowing us to retain the narrow size distribution and good crystallinity characteristic of this preparation method.

Initial work using these manganese precursors was promising: Mn was detected by EPR in all QD samples. However, when more careful purification was performed, no Mn signals were detected. Figure 1 displays an example of these results. $\mathrm{CdSe}$ nanocrystals were synthesized using $\mathrm{Mn}(\mathrm{CO})_{5} \mathrm{Me}$ as the dopant source; results for TCPMn and $\mathrm{MnMe}_{2}$ were exactly the same. Figure 1a shows the $9.44 \mathrm{GHz}$ EPR spectrum of this sample after size selective precipitation. The six line pattern is a signature of manganese hyperfine splitting (nuclear spin $I=$ $5 / 2$ ). However, after ligand exchange with pyridine, the sample was void of Mn signals (Figure 1b). This cap exchange process is known to remove more than $80 \%$ of the original TOPO

(12) (a) Wright, P. J.; Cockayne, B.; Cattell, A. F.; Dean, P. J.; Pitt, A. D. J. Cryst. Growth 1982, 59, 155-160. (b) Pain, G. N.; Rossouw, C. J.; Glanvill, S. R.; Rowe, R. S.; Dickson, R. S.; Deacon, G. B.; West, B. O. J. Cryst. Growth 1991, 107, 632-636.

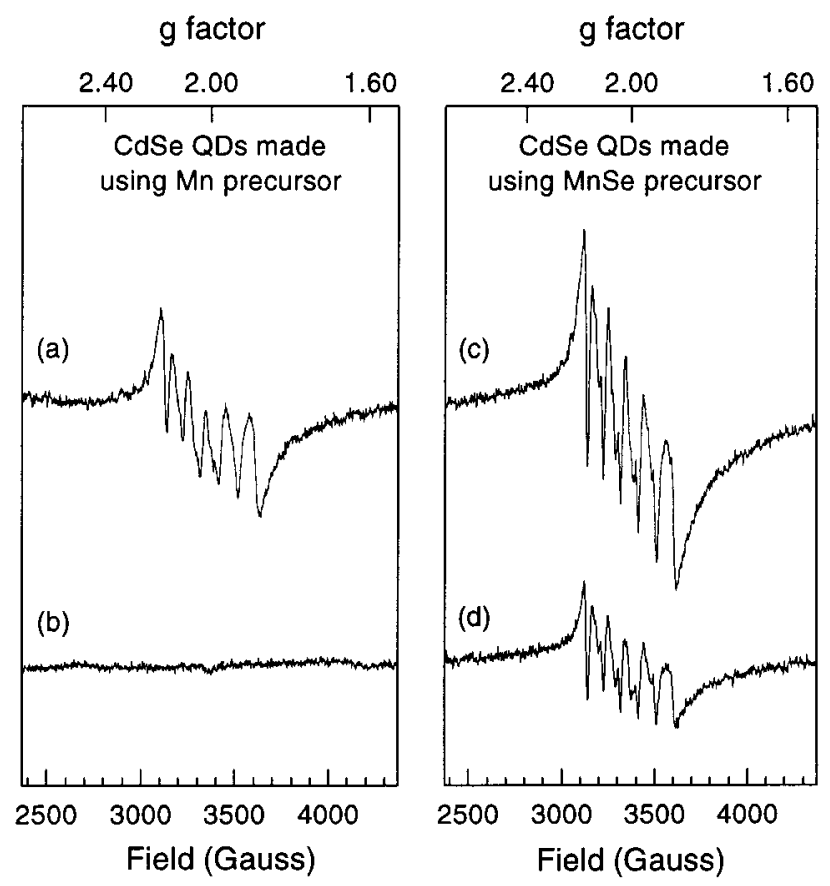

Figure 1. Low-temperature (5 K) EPR spectra of $40 \AA$ diameter CdSe quantum dots prepared using $(\mathrm{a}-\mathrm{b})$ a Mn only precursor and $(\mathrm{c}-\mathrm{d})$ the $\mathrm{Mn}_{2}(\mu \text {-SeMe })_{2}(\mathrm{CO})_{8}$ precursor. Before purification (a and c), both samples display the 6-line pattern characteristic of Mn. After pyridine cap exchange ( $b$ and $d$ ), only the sample prepared with the MnSe precursor shows any Mn signal. The hyperfine splitting in spectrum $\mathrm{d}$ is $83 \times 10^{-4} \mathrm{~cm}^{-1}$.

ligands, ${ }^{10 \mathrm{~b}}$ making this procedure the most effective way to purify doped QDs before elemental analysis. We believe that the initial Mn signals originated from loosely bound surface species or decomposition products not removed by size-selective precipitation.

To overcome the apparent unreactivity of manganese toward TOPSe, we synthesized an organometallic complex in which manganese and selenium were directly bonded. Such single source precursors have been described in the literature, and the decomposition of related compounds to manganese chalcogenides has been studied..$^{\mathrm{c}}$ Not only can the bulk material be made, but nanometer-sized $\mathrm{CdSe}^{13}$ and $\mathrm{CdMnSe}^{3 \mathrm{c}}$ have also been synthesized from single molecule precursors. Kanatzidis and co-workers employed a single source MnSe precursor (a manganese polychalcogenide anion) in a solution phase synthesis of $>125 \AA$ radius $\mathrm{Cd}_{1-x} \mathrm{Mn}_{x} \mathrm{Se}$ particles. Although the doping level range was all-inclusive, the particles were too large to exhibit quantum confinement effects, and the samples were generally polydisperse.

Doped CdSe nanocrystals were prepared using $\mathrm{Mn}_{2}(\mu$ $\mathrm{SeMe})_{2}(\mathrm{CO})_{8}$ as the manganese source (Figure 2). Figure $1 \mathrm{c}-\mathrm{d}$ displays the EPR spectra of a "MnSe"-doped sample before and after the same purification process used for the previous "Mn"doped sample. The Mn signal of this sample remains after size selective precipitation and surface ligand exchange with pyridine (Figure 1d).

Examination of the hyperfine splitting in the manganesedoped QDs provides information on the bonding characteristics of the dopant's local environment. The hyperfine splitting reported for $\mathrm{Mn}$ in bulk cubic CdSe is $62 \times 10^{-4} \mathrm{~cm}^{-1} .14$ The

(13) Trindade, T.; O’Brien, P.; Zhang, X. Chem. Mater. 1997, 9, 523530.

(14) Ludwig, G. W.; Woodbury, H. H. In Solid State Physics; edited by Seitz, F., Turnbull, D., Eds.; Academic Press: New York, 1962; Vol. 13, p 297. 


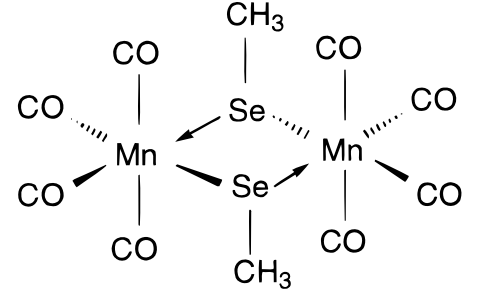

Figure 2. Precursor used to prepare manganese-doped CdSe nanocrystals.

hyperfine splitting of the Mn-doped QDs in Figure 1d is $83 \times$ $10^{-4} \mathrm{~cm}^{-1}$. This value was measured for all samples examined, and the explanation behind these larger splittings lies in the different bonding environments present near the surface of the QD versus the inner core. A report of the hyperfine splittings of $\mathrm{Mn}$ in $\mathrm{ZnS}$ nanocrystals found two separate signals, one with a hyperfine splitting $65 \times 10^{-4} \mathrm{~cm}^{-1}$ and the other $89 \times 10^{-4}$ $\mathrm{cm}^{-1} .{ }^{15}$ The smaller value, by comparison with the bulk material, was assigned to internal $\mathrm{Mn}$. The larger value, by analogy to DMS with $\mathrm{Mn}$ in more ionic hosts (such as $\mathrm{Li}_{2} \mathrm{O}$ ), was assigned to surface Mn. Since extensive washing experiments have confirmed that manganese is actually part of the inorganic core of the quantum dots, we conclude that the large hyperfine splitting indicates the dopant atoms generally reside near the more ionic surface layer of the nanocrystals. This conclusion is in agreement with etching experiments (see below) and a recent report which shows a progressive migration of manganese out of the nanocrystal lattice during annealing. ${ }^{16}$ The QDs appear to be effectively zone refining themselves. This result, in addition to the fact that TOPO is a particularly strong transition metal complexing agent, ${ }^{17}$ explains why highly doped samples could not be prepared by the present synthetic method.

Quantitative Determination of [Mn]. In light of the above findings, care was taken in preparing samples for all analytical measurements. $\mathrm{Mn}_{2}(\mu \text {-SeMe })_{2}(\mathrm{CO})_{8}$ was incorporated into the method described above to synthesize manganese-doped $\mathrm{CdSe}$ nanocrystals. These $\mathrm{Cd}_{1-x} \mathrm{Mn}_{x} \mathrm{Se}$ QDs were size selected three times, cap exchanged three times with pyridine, and brought back to TOPO/TOPSe passivation. In quantitative EPR experiments, Mn standard solutions were purposely mixed with undoped QDs. Normalizing the CdSe concentration between sample and standard minimized matrix effects and ensured that the microwave power absorbed by the dielectric background (solvent and CdSe host) was constant for the two solutions. We also found that the presence of the nanocrystals prevented aggregation of the Mn(II) cyclohexanebutyrate standard. Such clustering can cause enormous dipolar broadening of the EPR signal, an effect which has been described in the literature ${ }^{18}$ and can lead to serious errors in any quantitative measurement.

The importance of the cap exchange step in purifying these doped QDs is evident in Figure 3, which plots the normalized manganese concentration determined by EPR at selected points along the purification process. Note the break in the $x$-axis signifying the switch from size-selective precipitation to pyridine cap exchange. The more than 4-fold decrease in [Mn] with the first pyridine exchange confirms the presence of dopant atoms bound loosely to the nanocrystal surface. The dopant concentra-

(15) Kennedy, T. A.; Glasser, E. R.; Klein, P. B.; Bhargava, R. N. Phys. Rev. B 1995, 52, R14356-R14359.

(16) Levy, L.; Ingert, D.; Feltin, N.; Pileni, M. P. Adv. Mater. 1998, 10, $53-57$.

(17) Burgard, M.; Prevost, M. Phosphorus Sulfur 1983, 18, 319-322.

(18) (a) Allen, B. T.; Nebert, D. W. J. Chem. Phys. 1964, 41, 19831985. (b) Ross, R. T. J. Chem. Phys. 1965, 42, 3919-3922.

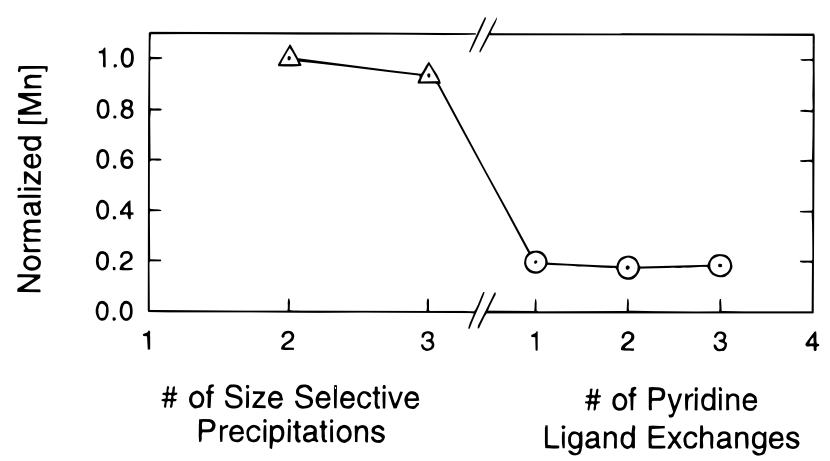

Figure 3. Variation of the normalized $[\mathrm{Mn}]$ in doped QDs with the extent of purification. Note the break in the $x$-axis indicating a switch from size-selective precipitation to pyridine ligand exchange.
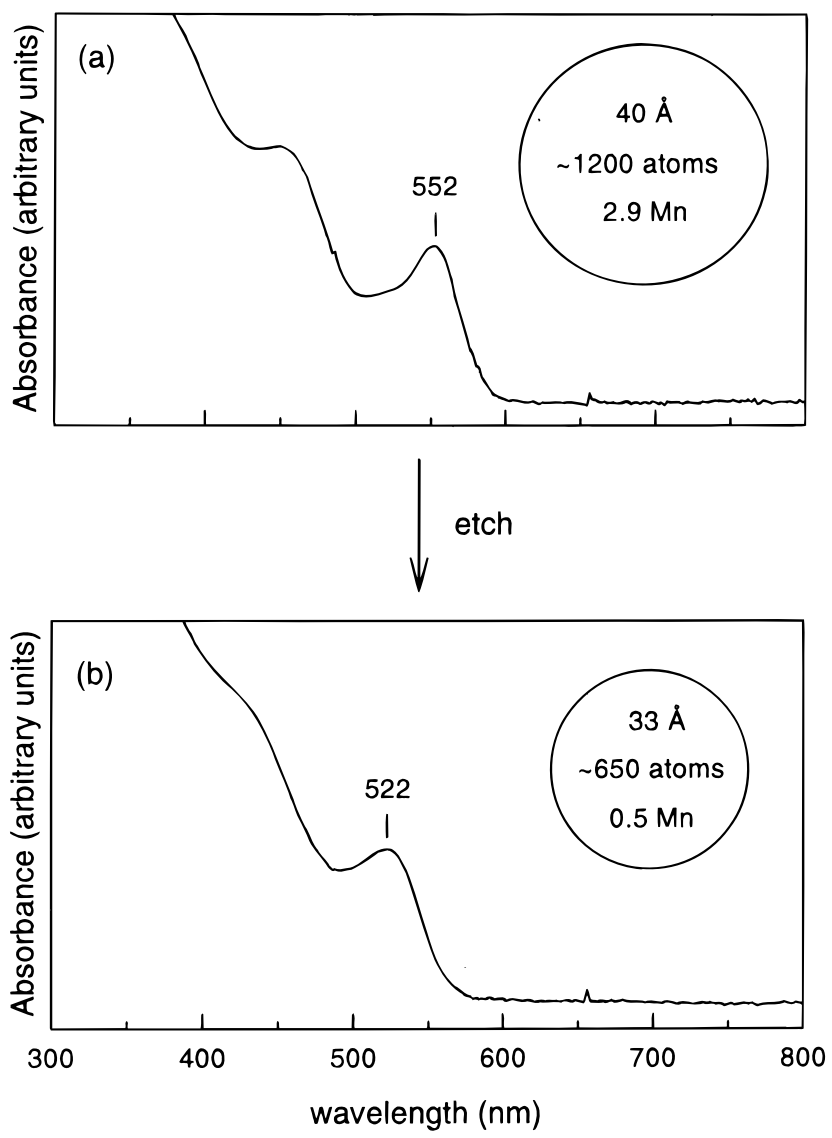

Figure 4. UV/visible absorption spectra of CdMnSe QDs (a) before and (b) after chemical etching with tripyrrolidinophosphine oxide.

tion is unchanged by subsequent pyridine treatments, consistent with the conclusion that any remaining manganese atoms must be inside the lattice.

To further exclude the possibility that the dopant is simply on the surface of the QDs, we chemically etched a sample of CdMnSe containing 2.9 Mn atoms per nanocrystal. Dispersing the sample in tripyrrolidinophosphine oxide (optical density at the first absorbing state $\sim 1.5$ in a $1 \mathrm{~cm}$ cell) and heating at 60 ${ }^{\circ} \mathrm{C}$ in air slowly shifts the band edge absorption of the QDs to shorter wavelengths, indicative of a smaller inorganic core. Figure 4 displays the UV/vis spectra from this experiment. Before etching (Figure 4a) the QDs are $40 \AA$ in diameter, contain approximately 1200 core atoms, and have an average of 2.9 $\mathrm{Mn}$ atoms per nanocrystal. After $2 \mathrm{~h}$ of stirring at $60{ }^{\circ} \mathrm{C}$ (Figure 4b) the QDs have shrunk to $33 \AA$ in diameter, contain approximately 650 core atoms, and now have $0.5 \mathrm{Mn}$ atoms 
Table 1. Compaison of [Mn] Determined by Two Independent Methods

\begin{tabular}{ccc}
\hline & \multicolumn{2}{c}{ Mn atoms per quantum dot } \\
\cline { 2 - 3 } sample & WDS & $\mathrm{EPR}^{a}$ \\
\hline 1 & $9.2 \pm 0.6$ & $4.9 \pm 0.6$ \\
2 & $8.6 \pm 0.7$ & $3.3 \pm 0.4$ \\
3 & $1.8 \pm 0.5$ & $1.2 \pm 0.1$ \\
\hline
\end{tabular}

${ }^{a}$ Experimental uncertainties reflect the precision associated with measuring [Mn]. The EPR experiment is complicated by the fact that, unlike WDS, it does not directly measure the concentration of quantum dots in a sample. Using data from other syntheses of similar nanocrystal size (42 A diameter), we assume an $85 \%$ yield of CdSe. If doping altered the growth and the true yield is half that, then the EPR values would have to be multiplied by 2 .

per nanocrystal. Two important results come from this simple experiment. First, the presence of $\mathrm{Mn}$ in the etched sample confirms that the inorganic core of some nanocrystals is doped. Second, if the Mn distribution in the nanocrystal lattice were random, then the dopant concentration in Figure $4 \mathrm{~b}$ would scale with the overall decrease in number of atoms. Since the Mn concentration falls by $83 \%$ upon removal of about half of the outer atoms, most of the dopant atoms reside near the quantum dot surface. This finding agrees with the EPR results (discussed above) which also indicate that the Mn is near the surface.

Quantitative analysis by both EPR and WDS experiments on the same Mn-doped CdSe samples (42 A diameter) is shown in Table 1. The uncertainties reflect the precision to which the Mn concentrations could be determined. However, there is a systematic error associated with the EPR measurement because this technique, unlike WDS, does not directly measure a value for the concentration of QDs. Precise values for the sizedependent extinction coefficient of CdSe QDs do not exist. Since the synthesis of similar sized CdSe produced an $85 \%$ yield of material, we assume this value in our calculations. However, if doping alters the growth and the true yield is half that value, then the EPR concentrations would have to be multiplied by a factor of 2. Whether or not such a correction is necessary, the important point is that these two very different experiments agree to within a factor of 2 or 3 . Given the uncertainty involved in determining the concentration of QDs in the EPR samples, this experiment should be viewed as a rough check of the accuracy of the WDS measurements. Most importantly, with the proper precautions and sufficient attention to the surface chemistry of these materials, reliable values can be obtained even for very low dopant concentrations.

Precise measurements of dopant concentrations in bulk DMS can also be made by powder X-ray diffraction, since the positions of the X-ray peaks have been found to vary linearly with concentration (Vegard's law). ${ }^{1}$ However, due to finite size broadening of X-ray features and the very low doping levels in our particles, we could not observe a difference between the wide-angle X-ray spectra of undoped and doped QDs.

${ }^{113}$ Cd MAS NMR. Paramagnetic centers can significantly affect NMR spectra, leading to line shifts, line broadening, and shorter longitudinal relaxation times. ${ }^{19}$ Figure 5 displays the results from MAS solid-state NMR experiments $\left[\delta\left(\mathrm{Cd}\left(\mathrm{NO}_{3}\right)_{2}\right)\right.$ $=0 \mathrm{ppm}]$. Estimated spin lattice relaxation times are given at the right of each spectrum. Note the long $T_{1}$ value of $134 \mathrm{~s}$ for bulk CdSe powder (Figure 5a). In the case of TOPO-capped CdSe QDs (Figure 5b), the line has broadened considerably and the ${ }^{113} \mathrm{Cd}$ relaxation time has increased beyond the bulk value. Both these effects lead to a lower signal-to-noise ratio, which

(19) Drago, R. S. Physical Methods for Chemists; Saunders College Pub.: Ft. Worth, 1992; pp 500-558.

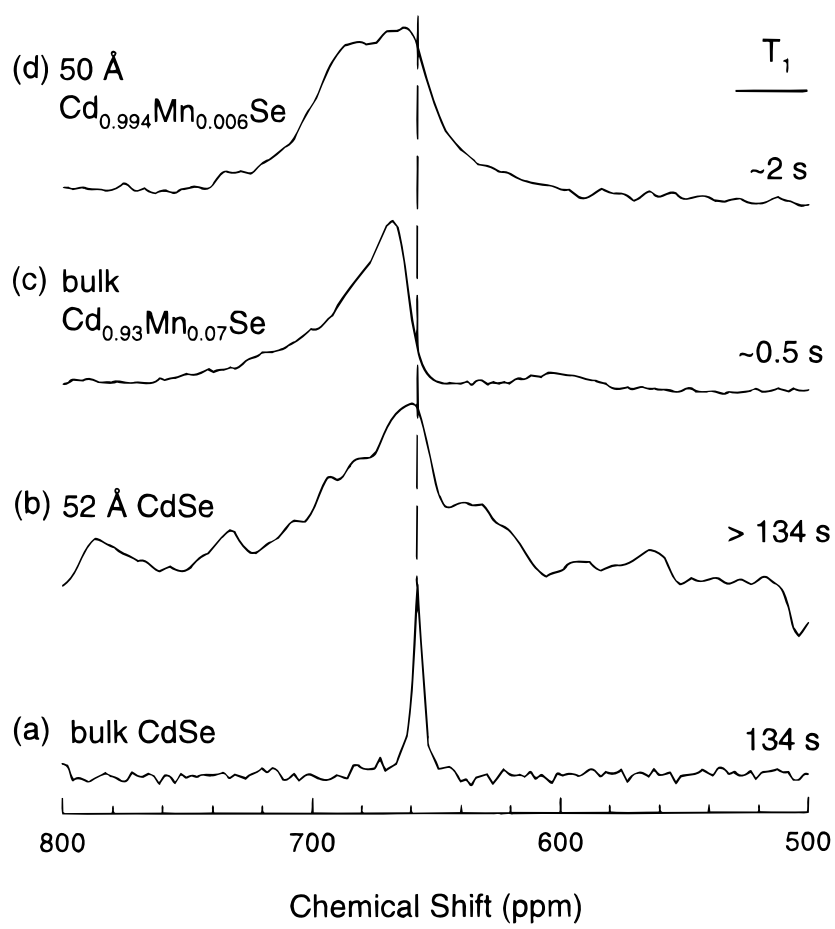

Figure 5. Solid-state NMR spectra of (a) bulk CdSe, (b) $52 \AA$ diameter CdSe QDs, (c) bulk Cd $\mathrm{Cd}_{0.93} \mathrm{Mn}_{0.07} \mathrm{Se}$, and (d) $50 \AA \mathrm{Cd}_{0.994} \mathrm{Mn}_{0.006} \mathrm{Se}$ QDs. Smaller chemical shift values correspond to higher field strengths.

is evident in the spectrum even though the total accumulation time was $24 \mathrm{~h}$. For the CdSe QDs dispersion in the line shape is due to a distribution of chemical shifts present in a nanocrystal: roughly $22 \%$ of the atoms lie on the surface of a $52 \AA$ diameter spherical CdSe cluster. A chemical shift dispersion due to this large surface-to-volume ratio has also been reported in the ${ }^{77} \mathrm{Se}$ solution-phase NMR of $\mathrm{CdSe} \mathrm{QDs}^{20}$ and in the ${ }^{113} \mathrm{Cd}$ solution-phase NMR of molecular $\left[\mathrm{S}_{4} \mathrm{Cd}_{10}(\mathrm{SPh})_{16}\right]^{4-}$ clusters. $^{21}$

The effects of the manganese dopant are evident in the top two spectra of Figure 5. The NMR spectrum of bulk $\mathrm{Cd}_{0.93^{-}}$ $\mathrm{Mn}_{0.07} \mathrm{Se}$ powder in Figure 5c displays both a shift and an asymmetric line shape relative to undoped bulk CdSe in Figure 5a. The distribution in chemical shifts reflects the distribution of $\mathrm{Cd}$ atoms around the $\mathrm{Mn}$ center and is in excellent agreement with the line shape reported for the ${ }^{113} \mathrm{Cd}$ NMR line in bulk $\mathrm{Cd}_{1-x} \mathrm{Mn}_{x}$ Te. $^{22}$ The experimental $T_{1}$ value of $\sim 500 \mathrm{~ms}$ is comparable to other DMS alloys $\mathrm{Cd}_{1-x} \mathrm{Co}_{x} \mathrm{Se}(<600 \mathrm{~ms})^{23}$ and $\mathrm{Cd}_{1-x} \mathrm{Fe}_{x} \mathrm{Te}(793 \mathrm{~ms}) .{ }^{22}$ Note that the introduction of $\mathrm{Mn}$ has decreased $T_{1}$ by more than 2 orders of magnitude. In agreement with ref 23 no rotational sidebands were observed from a transferred hyperfine interaction between the unpaired electrons of manganese and the ${ }^{113} \mathrm{Cd}$, indicating that both bulk and nanocrystal samples have qualitatively similar behavior.

The effects seen in the spectrum of bulk CdMnSe (Figure 5c) are also present in the doped QD spectrum (Figure 5d), providing further evidence that manganese atoms are actually substituted in the host lattice. This $\mathrm{Cd}_{0.994} \mathrm{Mn}_{0.006} \mathrm{Se}$ sample contains $\sim 7 \mathrm{Mn}$ atoms per QD. The most substantial effect of the $\mathrm{Mn}$ is on the spin lattice relaxation time, which has decreased

(20) Thayer, A. M.; Steigerwald, M. L.; Duncan, T. M.; Douglass, D C. Phys. Rev. Lett. 1988, 60, 2673-2676.

(21) Dance, I. G. Aust. J. Chem. 1985, 38, 1745-1755.

(22) Gavish, M.; Vega, S.; Zamir, D. Phys. Rev. B 1993, 48, $2191-$ 2199.

(23) Ladizhansky, V.; Faraggi, A.; Lyahovitskaya, V.; Vega, S. Phys. Rev. B 1997, 56, 6712-6718. 
from a value greater than $134 \mathrm{~s}$ in undoped QDs to $\sim 2 \mathrm{~s}$ in the doped sample. A shift and broadening in the presence of the paramagnet were also observed. The NMR spectrum (not shown) of CdSe quantum dots in which a manganese salt was simply mixed into the sample (and therefore extrinsic to the nanocrystal core) did not display these features. In the QD spectrum (Figure 5d) a well-defined shoulder is visible. This feature is likely a combination of two effects, the first being the distribution of cadmium nuclei positions with respect to the manganese dopant, which leads to the asymmetry seen in Figure 5c. The second effect is the chemical shift dispersion (discussed above) associated with surface versus core sites in the quantum dot. In fact, a low-field shoulder may already be present in the spectrum of undoped CdSe QDs (Figure 5b).

Optical Spectroscopy. Previous experiments by Nirmal and co-workers have documented the effects of strong external magnetic fields (up to $10 \mathrm{~T}$ ) on both the fluorescence line narrowed (FLN) spectra and the luminescence lifetimes. ${ }^{24}$ To see if the manganese atom has the same effect as an external field, we perform FLN spectroscopy on a doped nanocrystal sample. We also expected to see a decrease in the fluorescence lifetime, but data from lifetime decay experiments were inconclusive, most likely because the extensive cap exchange procedure used on these samples has affected the photoluminescence quantum yield. We concentrate below on the FLN and corresponding photoluminescence excitation (PLE) experiments.

Bulk $\mathrm{Cd}_{1-x} \mathrm{Mn}_{x}$ Se displays only band edge luminescence for $0 \leq x \leq 0.45$. $^{25}$ Similarly, for all CdMnSe nanocrystal samples synthesized, only band edge CdSe luminescence was observed.

Figure 6 displays raw FLN, PLE, and PL spectra for $(\mathrm{a}-\mathrm{b})$ undoped and $(\mathrm{c}-\mathrm{d})$ doped $26 \AA$ diameter CdSe quantum dots (about 320 core atoms). WDS determined the manganese concentration of the doped sample to be 1.5 atoms per quantum dot. The complete study (available in the Supporting Information) encompasses five temperatures: $15,10,5,3$, and $1.75 \mathrm{~K}$. For brevity, we show only the 10 and $1.75 \mathrm{~K}$ data. At each temperature we excite at five different wavelengths (indicated by vertical lines on the full fluorescence spectra in Figure 6, panels a and b) and collect the corresponding FLN emission spectra, seen on the left side of each graph. Similarly, monitoring the emission at the maximum of the zero LO phonon line (ZPL) while scanning through the excitation produces the PLE spectra seen on the right side of the graphs. In this way we probe the residual distribution still present in these optically size selected subsets of the sample.

Subtle qualitative differences are present in the raw data: at $1.75 \mathrm{~K}$ the FLN spectrum of the doped QDs (Figure 6d) appears to show an increase in the relative intensity of the one-phonon line (1PL) with respect to the ZPL in comparison to the undoped QDs (Figure 6b). This increase is important because FLN experiments on QDs in a magnetic field show the same temperature dependence of the 1PL/ZPL ratio (also known as the Huang-Rhys parameter $S$ ). ${ }^{24}$ A priori, however, it is difficult to say whether these changes are due to probing different sizes in each sample or from sample-to-sample variations in the residual size distribution. In addition, the PLE data are contaminated with FLN information (and vice versa) due to the residual size distribution. To obtain a more quantitative description of temperature-dependent changes in the optical properties of the two samples, we employ a fitting routine to extract effective QD absorption and emission line shapes (so-called

(24) Nirmal, M.; Norris, D. J.; Kuno, M.; Bawendi, M. G.; Efros, A. L.; Rosen, M. Phys. Rev. Lett. 1995, 75, 3728-3731. K80.
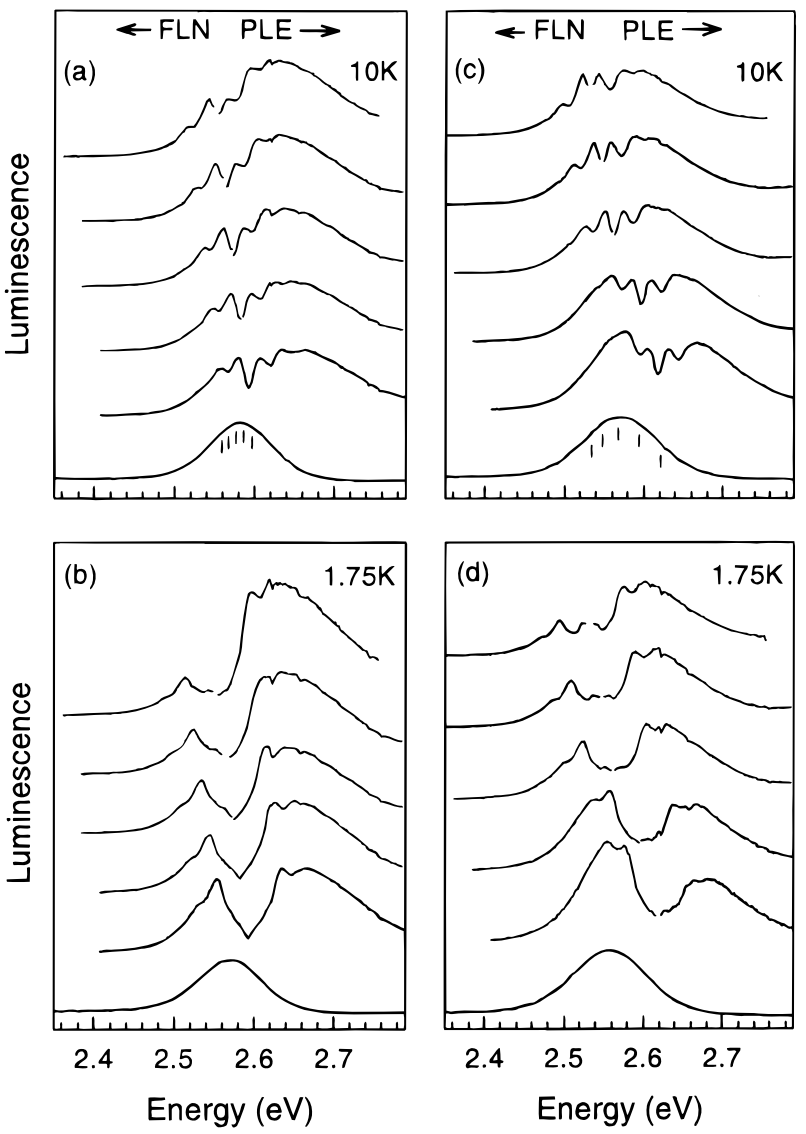

Figure 6. Normalized FLN and PLE experimental data for $(a-b)$ undoped and $(\mathrm{c}-\mathrm{d}) \mathrm{Mn}$-doped CdSe QDs. For each different temperature we collect five different FLN-PLE pairs to probe the residual distribution in the sample. The excitation energies for the FLN experiments are given by the vertical lines on the full PL in panels a and $\mathrm{c}$, with the same excitation positions used in panels $\mathrm{b}$ and $\mathrm{d}$, respectively.

"single dot" line shapes). ${ }^{26}$ These effective line shapes are simply the ones required to best reproduce the experimental data. They still contain inhomogeneities inherent in the FLN/ PLE experiment and are not the actual line shapes of a single quantum dot. ${ }^{27,28}$

The fitting procedure is based upon a theoretical fine structure model, ${ }^{29}$ also known as the dark exciton model because it predicts the lowest excited state to be an optically dark exciton with angular momentum projection \pm 2 . By taking into account splitting of the 8-fold degenerate exciton ground state by the electron-hole exchange interaction and intrinsic nanocrystal asymmetry, this model has successfully described the results of many optical experiments on CdSe quantum dots, including hole burning, ${ }^{30}$ FLN/PLE, ${ }^{24,10 b}$ and magnetic circular dichroism. ${ }^{31}$ Using the states dictated by this theory, the FLN, PLE, and PL data are fit simultaneously by convoluting an effective

(26) (a) Norris, D. J.; Efros, A. L.; Rosen, M.; Bawendi, M. G. Phys Rev. B 1996, 53, 16347-16354. (b) Kuno, M. Ph.D. Thesis, Massachusetts Institute of Technology, 1998; pp 229-246.

(27) Nirmal, M.; Dabbousi, B. O.; Bawendi, M. G.; Macklin, J. J.; Trautman, J. K.; Harris, T. D.; Brus, L. E. Nature 1996, 383, 802-804. (28) Empedocles, S. A.; Norris, D. J.; Bawendi, M. G. Phys. Rev. Lett. 1996, 77, 3873-3876.

(29) Efros, A. L.; Rosen, M.; Kuno, M.; Nirmal, M.; Norris, D. J.; Bawendi, M. Phys. Rev. B 1996, 54, 4843-4856.

(30) Norris, D. J.; Sacra, A.; Murray, C. B.; Bawendi, M. G. Phys. Rev. Lett. 1994, 72, 2612-2615.

(31) Kuno, M.; Nirmal, M.; Bawendi, G.; Efros, A.; Rosen, M. J. Chem. Phys. 1998, 108, 4242-4247. 


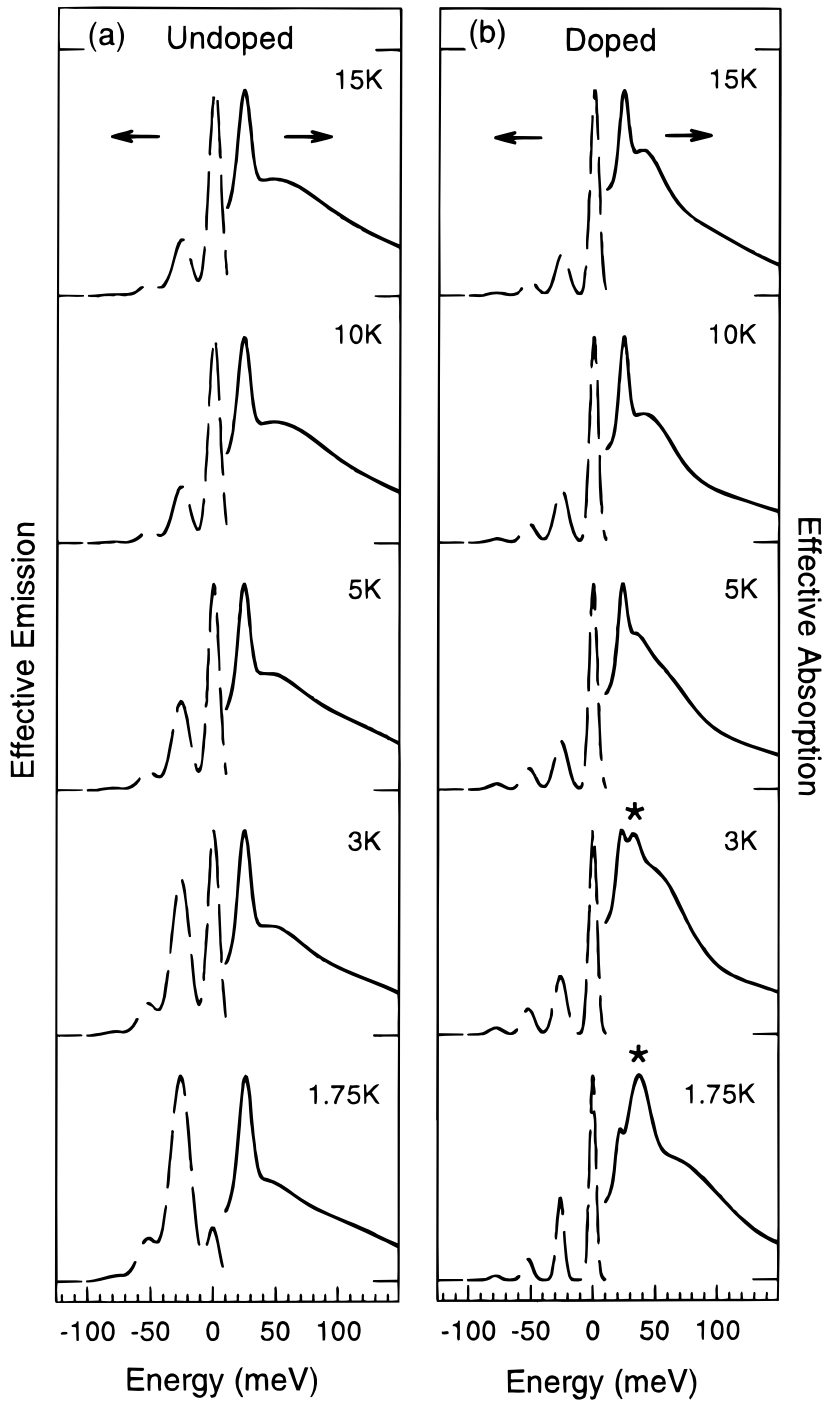

Figure 7. Temperature evolution of effective "single dot" emission (dashed lines) and absorption (solid lines) line shapes for (a) undoped and (b) doped CdSe QDs. The asterisks in panel b denote the position of the extra state which had to be added to achieve satisfactory fits to the data.

emission line shape, an effective absorption line shape, and a normalized Gaussian distribution. In all cases, very good fits were obtained (see the Supporting Information). We focus on the effective "single dot" line shapes, shown in Figure 7 for (a) undoped and (b) doped QDs over the entire temperature range studied.

The fitting results for the undoped CdSe QDs (Figure 7a) show basically no change in the effective single dot absorption (solid line) with temperature. In the effective single dot luminescence (dashed line), however, the ratio of the intensity of the 1PL to the ZPL increases dramatically as the temperature falls. The low-temperature $1.75 \mathrm{~K}$ spectrum is qualitatively explained by noting that the emitting state is formally spin forbidden: phonon assisted emission accounts for the enhanced 1PL and large Huang-Rhys parameter. As the temperature is raised, optically bright character from a nearby state mixes with the dark exciton, and emission from the ZPL dominates. The exact mechanism by which this mixing occurs is still unknown, although nonadiabaticity of the nanocrystal exciton-phonon system has recently been proposed as an explanation. ${ }^{32}$

(32) Fomin, V. M.; Gladilin, V. N.; Devreese, J. T.; Pokatilov, E. P.; Balaban, S. N.; Klimin, S. N. Phys. Rev. B 1998, 57, 2415-2425.
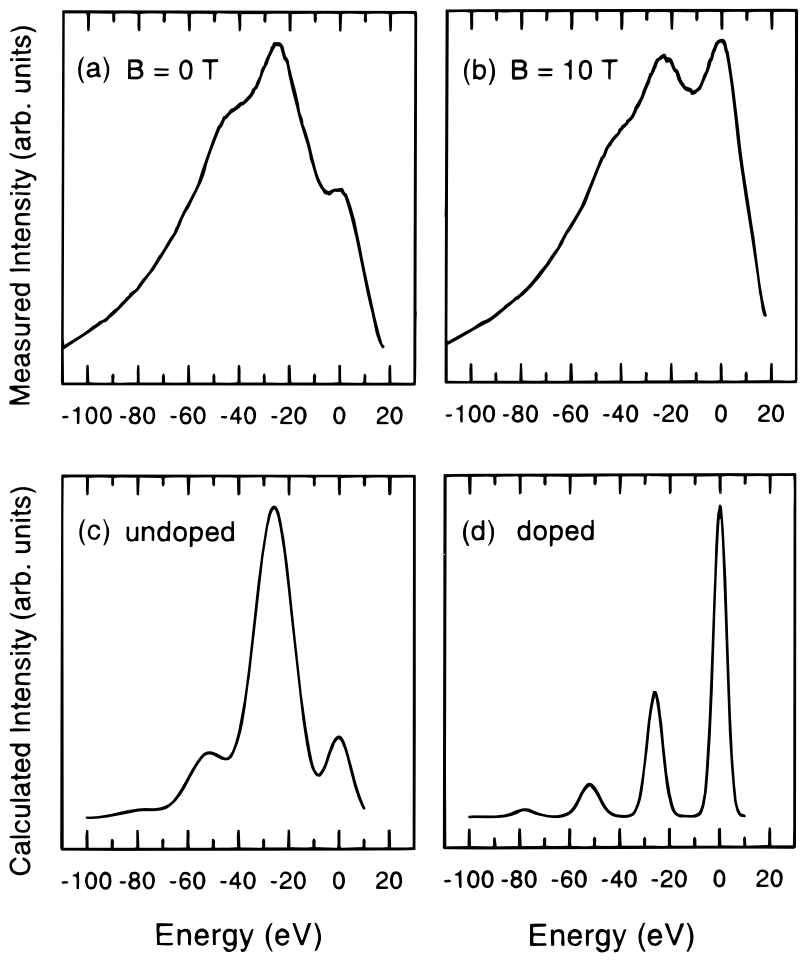

Figure 8. Comparison of raw FLN data (top row) with calculated "single dot" line shapes for $26 \AA$ diameter CdSe QDs under different conditions: raw FLN data in (a) zero applied magnetic field and (b) $10 \mathrm{~T}$ field; calculated emission line shapes for (c) undoped QDs and (d) CdSe doped with 1.5 Mn atoms per QD. The raw FLN data are taken from ref 24. All graphs represent data taken or simulated at 1.75 K. Qualitatively, both applying an external magnetic field and doping with a paramagnetic atom have the same effect on the QD luminescence.

Doped QDs behave very differently from undoped QDs. The effective single dot line shapes for the doped sample (Figure $7 b)$ display significant differences from the undoped sample in both emission and absorption. As the temperature decreases, the emission (dashed lines) shows no increase in the HuangRhys parameter, unlike the undoped sample. The 1PL/ZPL ratio is fairly constant over the full range of temperatures. These results show essentially the same behavior as undoped QDs in an external magnetic field. ${ }^{24}$ The exchange field arising from the interaction of the manganese $d$ electrons and the photogenerated electron and hole can be quite large. Estimates of the magnetic moment of a bound magnetic polaron (a carrier spin "dressed" by surrounding aligned impurity spins) are as high as $50 \mu_{\mathrm{B}}{ }^{33}$ Such a moment should produce very strong effective local magnetic fields, on the order of a few Tesla for a $5 \AA$ distance. For any experiment that aims to split the energy levels of the quantum dot with an external magnetic field, an equivalent experiment can be performed simply by doping the quantum dot. This is a key result of this paper. The fact that doping with $\mathrm{Mn}$ is equivalent to placing the QDs within a magnetic field is illustrated in Figure 8, which displays FLN spectra and calculated line shapes of CdSe QDs at $1.75 \mathrm{~K}$ under four conditions. Note that the top two graphs are experimental data from ref 24 , whereas the bottom two graphs are calculated single dot emission line shapes from the present work. Due to differences in both experimental setup and data collection method, a direct quantitative comparison between the two experiments' results (either raw or calculated spectra) is not possible. However, a qualitative discussion of Figure 8 supports

(33) Wolff, P. A. In Semiconductors and Semimetals; Furdyna, J. K.; Kossut, J., Eds.; Academic Press: Boston, MA, 1988; Vol. 25, p 444. 


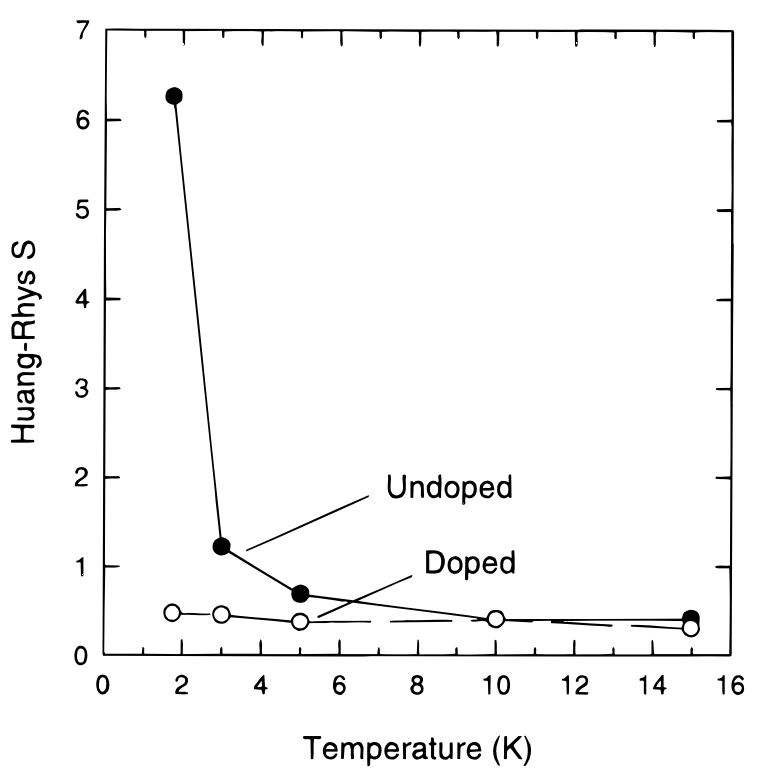

Figure 9. Temperature dependence of the Huang-Rhys parameter $S$ (1PL/ZPL ratio) from the effective line shapes of Figure 7.

the previous assertion. The top two graphs of Figure 8 are experimental FLN spectra of $26 \AA \mathrm{CdSe}$ (a) in zero field and (b) in a $10 \mathrm{~T}$ field. Components of the external field perpendicular to the nanocrystal $c$-axis act as a perturbation to the electronic structure, effectively mixing the formally dark \pm 2 emitting state with the neighboring $\pm 1^{\mathrm{L}}$ exciton, making fluorescence from the ZPL the dominant pathway for radiative relaxation. ${ }^{24}$ Increasing the magnetic field strength increases the intensity of the ZPL. The bottom two graphs of Figure 8 are calculated line shapes of (c) undoped CdSe and (d) Mn-doped CdSe. The act of doping the CdSe has increased the ZPL intensity at the expense of the 1PL. Doping the QD has produced the same effect as a magnetic field.

As in the emission, the effective single dot absorption spectra (solid lines) of the doped sample (Figure 7b) show different features from the undoped sample (Figure 7a). To obtain acceptable fits to the raw data, it was necessary to add an extra state to the standard 3 optically active levels used to model the absorption in the case of the undoped QDs. This new state manifests itself as the feature marked with an asterisk in Figure 7b. We speculate that this transition, which has not been observed before, is one of the five fine structure states predicted by theory. Based upon its position relative to the lowest absorbing state (the $\pm 1^{\mathrm{L}}$ exciton), this new feature is likely the formally dark $0^{\mathrm{L}}$ exciton. This new feature appears an average of $12.4 \mathrm{meV}$ away from the lowest absorbing state, whereas the calculated spacing is $5.4 \mathrm{meV}$.

Figure 9 shows the temperature dependence of the HuangRhys parameter extracted from Figure 7 and reiterates the differences observed between undoped and doped nanocrystals. The undoped sample shows a dramatic upturn in the integrated phonon ratio at temperatures near $3 \mathrm{~K}$. The doped sample shows a relatively constant Huang-Rhys parameter over the full range of temperatures, just as undoped samples do in an external magnetic field. This behavior is consistent with the mixing of fine structure states through the unaligned exchange field of the paramagnetic dopant.

Figure 10 shows the behavior of the relative oscillator strength for the lowest absorbing state $\left( \pm 1^{\mathrm{L}}\right)$ of doped and undoped samples as a function of temperature. It is this $\pm 1^{\mathrm{L}}$ state that the FLN experiment excites into. The graph plots the results of the fitting procedure. Mixing of the optically active $\pm 1^{\mathrm{L}}$ state

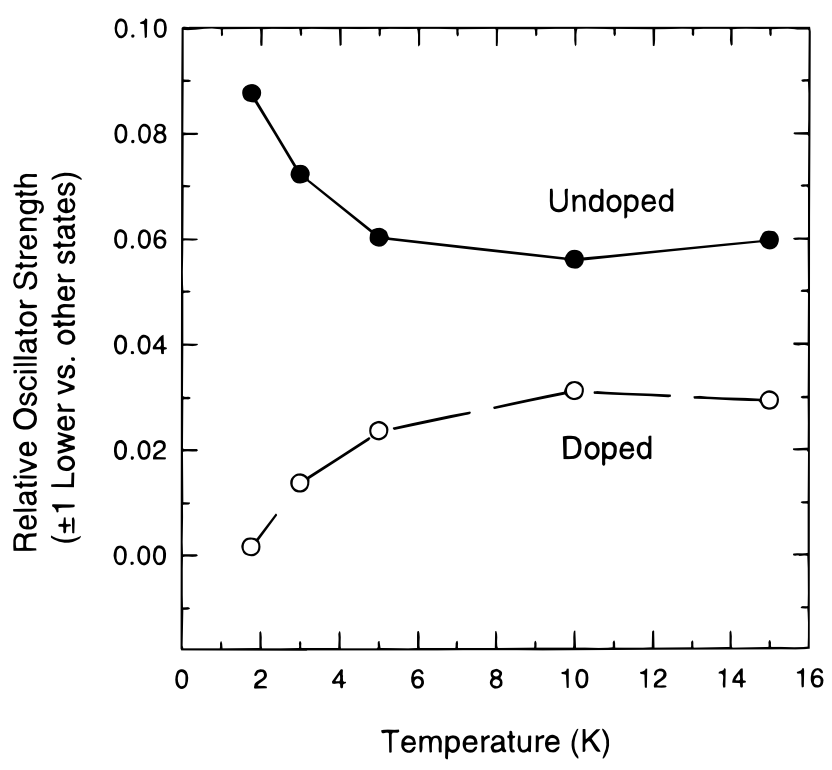

Figure 10. Relative oscillator strength of the $\pm 1^{\mathrm{L}}$ state with respect to all other optically active fine structure states. The decrease in oscillator strength seen in the doped sample at lower temperatures is due to magnetic field induced mixing with nearby optically dark states.

with surrounding optically dark states decreases as temperature decreases. Less mixing implies more bright character for the $\pm 1^{\mathrm{L}}$ state, which explains the increase in the relative oscillator strength of undoped QDs with decreasing temperature.

In contrast to the undoped sample, the doped sample has $\pm 1^{\mathrm{L}}$ level oscillator strength which decreases with temperature. This behavior implies that the $\pm 1^{\mathrm{L}}$ state is being mixed with an energy state that is optically dark. The FLN data have shown that the effective magnetic field of the dopant mixes quantum dot electronic states down to $1.75 \mathrm{~K}$. The temperture dependence of the relative oscillator strength of the $\pm 1^{\mathrm{L}}$ state is consistent with the FLN data.

\section{Conclusion}

We have successfully synthesized manganese-doped CdSe nanocrystal colloids whose surfaces are passivated with TOPO organic ligands. The organometallic complex $\mathrm{Mn}_{2}(\mu \text {-SeMe })_{2}-$ $(\mathrm{CO})_{8}$ was used as the dopant source. Quantitative EPR and WDS measurements on manganese-doped nanocrystals are in agreement. Dopant levels of $\sim 1 \mathrm{Mn}$ atom per QD can be reproducibly achieved. Both a simple etching experiment and EPR hyperfine splitting show that the Mn dopant is distributed mainly near the surface of the $\mathrm{CdSe}$ lattice. The ${ }^{113} \mathrm{Cd}$ solidstate NMR experiments reveal a much smaller $T_{1}$ relaxation time and a paramagnetic shift in the doped samples, consistent with manganese having substituted somewhere inside the nanocrystal lattice.

Optical experiments show that doped QDs behave like undoped QDs in an external magnetic field. The behavior of doped QDs is consistent with the presence of an exchange field that is due to the interaction of the electron/hole spin with the paramagnetic impurity and that has a component perpendicular to the crystallite $c$-axis. In the effective "single dot" emission spectra of the doped QDs, the Huang-Rhys parameter remains nearly constant with temperature, a behavior that has also been observed for undoped QDs in an external magnetic field. At very low temperatures $(<3 \mathrm{~K})$ a new state emerges in the effective absorption spectra. The position of this transition suggests that it is the optically dark $0^{\mathrm{L}}$ exciton predicted by a recent theoretical model describing CdSe QDs. PLE of these 
doped CdSe QDs provides new spectroscopic information which reveals a previously unobserved, but theoretically predicted, fine structure state. The simple synthetic scheme presented in this paper not only outlines a new route to an interesting material but also provides a powerful method for performing high magnetic field experiments on nanometer scale systems.

Acknowledgment. The authors gratefully acknowledge valuable discussions with Al. L. Efros and M. Nirmal. We thank N. Chatterjee for WDS measurements, M. Pravia for assistance obtaining ${ }^{113} \mathrm{Cd}$ NMR spectra, and A. Sacra and C. A. Leatherdale for lifetime measurements. F.V.M. and M.K. received support from an NSF Environmental Training Grant and the Corning Foundation. This work was supported in part by the David and Lucille Packard Foundation, the Sloan Foundation, the NSF-MRSEC program (NSF-DMR-98-08941), and the Harrison Spectroscopy laboratory (NSF-CHE-97-08265).

Supporting Information Available: Table of CdSe $\lambda_{\max }$ versus size; FLN, PLE, and PL spectra (experimental and calculated) of undoped and doped QDs at 15, 10, 5, 3, and 1.75 $\mathrm{K}(\mathrm{PDF})$. This material is available free of charge via the Internet at http://pubs.acs.org.

JA991249N 